\title{
Melatonin attenuates RVLM neuro-inflammation and sympathetic activation in stress-induced hypertension rats.
}

\author{
Zhang Shutian*
}

Clinical Medicine (Eight-year Program), Shanghai Medical College, Fudan University, 138 Yi xue yuan Road, Shanghai, China, 200032

*Corresponding author: Shutian Zhang, E-mail address: stzhang15@ fudan.edu.cn

Abstract Background: Hypertension is the cardiovascular syndrome with the highest morbidity and mortality worldwide. Hypertension caused by various stress factors is called stress-induced hypertension (SIH). The rostral ventrolateral medulla (RVLM) "neuroinflammatory-sympathetic overactivation" is involved in SIH formation. Melatonin has anti-inflammatory, anti-oxidant and blood pressure lowering effects. The present study is to explore the antihypertensive effects and mechanism of central melatonin which based on microglia derived neuroinflammation. Methods: Stress-induced hypertension (SIH) was induced by electric foot-shock stressors with noise interventions in rats. Melatonin $(0.01,0.1,1 \mathrm{mmol} / \mathrm{L})$ was administered to RVLM and then blood pressure (BP) and serum norepinephrine (NE) were monitored to reflect sympathetic vasomotor activity in SIH rats. Excitatory neurotransmitter (Glutamate) and inhibitory neurotransmitter [ $\gamma$-aminobutyric acid (GABA)] were measured using ELISA kits. Markers of microglia M1 polarization (CD86) and pro-inflammatory cytokines (PICs (IL-1 $\beta$, TNF- $\alpha$ )) expression in the RVLM were measured by RTqPCR. Results: (1) Stress induced increase in blood pressure and serum NE concentration; RVLM microinjection melatonin attenuated the elevation of blood pressure and increasing of plasma NE in SIH rats in a dose-dependent manner. (2) The expression of CD86, PICs (IL-1 $\beta$, TNF- $\alpha$ ) and c-fos were increased in SIH rats; RVLM injection melatonin attenuated RVLM neuroinflammation and its effect is concentration dependent. (3). Stress induced increase in glutamate concentration in RVLM; RVLM injection melatonin reduced glutamate level and increased GABA level in SIH rats in a concentration-dependent manner. Conclusion: RVLM injection of melatonin inhibits M1 polarization and has anti-hypertensive effects. Melatonin reduce M1 polarization in microglia might be a novel target and new strategy for anti-stress induced-hypertension.

Keywords: Stress; Hypertension; Microglia; Neuroinflammation; Sympathetic overactivation 


\section{Introduction}

Hypertension (HTN) is the major cause of morbidity and mortality throughout the world. More than half of the clinical cases of stroke and ischemic heart disease can be attributable to hypertension. Various stress factors contribute to stress-induced hypertension $(\mathrm{SIH})$ development. However, it is uncertain exactly how stress might contribute to the pathogenesis of SIH. It is well established that microglia-derived neuroinflammation in cardiovascular center rostral ventrolateral medullar (RVLM) contribute to sympathetic overactivation and hypertension development $[1,2]$. Chronic stress cause potent glial cell activation and release of immune and proinflammatory mediators in RVLM, which leads to persistent low-grade neuroinflammation [3, 4]. Melatonin exhibits various pharmacological activities such as anti-inflammatory, anti-aging, hypolipidemic, hypotensive, cardio-protective and neuro-protective effects. The decrease of melatonin is associated with the increase of blood pressure and the occurrence of non-dipper blood pressure rhythm [5, 6]. We previously reported that exogenous administration of melatonin in the pre-hypothalamic region (AHA) of SIH rats produced hypotensive effects. However, none of the previous studies have explored the antihypertensive targets of central melatonin against SIH. In this study, we observed that melatonin can suppress stress-induced RVLM microglial M1 polarization and neuroinflammation, in turn, inhibiting sympathetic activation in SIH rats.

\section{Methods}

\subsection{Reagents}

A total of 36 male SD rats $(220 \mathrm{~g} \pm 10 \mathrm{~g})$ were provided by the experimental animal center of Fudan University and randomly divided into 6 groups: blank control group (Ctrl), stress group (SIH), stress+artificial cerebrospinal fluid control group $(\mathrm{SIH}+\mathrm{aCSF}), \mathrm{SIH}+0.01 \mathrm{~mm}$ melatonin intervention group [SIH+Mel $(0.01 \mathrm{mM})]$, $\mathrm{SIH}+0.1 \mathrm{~mm}$ melatonin intervention group $[\mathrm{SIH}+\mathrm{Mel}(0.1 \mathrm{mM})]$, and $\mathrm{SIH}+1 \mathrm{mM}$ melatonin intervention group $[\mathrm{SIH}+\mathrm{Mel}(1 \mathrm{mM})]$, with 6 rats in each group. All experiments were performed in accordance with the Animal Care Committee of Shanghai Medical College of Fudan University, and the National Institutes of Health Guide for the Care and Use of Laboratory Animals, and every effort was made to minimise the number of animals used, as well as any pain and discomfort. Melatonin was purchased from SIGMA. RNAeasy ${ }^{\mathrm{TM}}$ cell RNA extraction kit (centrifugal 
column) was purchased from Beyotim (Nanjing, PRC). Primers of objective genes and internal reference gene were synthesized by Sangon (Shanghai, PRC). Quant one step qRT-PCR Kit (SYBR Green) (FP303) reaction Kit was purchased from Qiagen (Beijing, PRC). Rabbit anti-rat CD86, TNF- $\alpha$, IL-1 monoclonal antibodies were purchased from Abcam. Antibody-tubulin was purchased from Santa cruz. The study was approved by Shanghai Medical college of Fudan University with the approval number: QF1703.

\subsection{Preparation of rat model of stress induced hypertension}

The rat model of stress induced hypertension was prepared with reference to our published paper [7]. The rats were stimulated by low frequency and low voltage alternating current (ac). The rats were stimulated twice a day for $2 \mathrm{~h}$ for 15 days. The current intensity of electrical stimulation is $2 \sim 4 \mathrm{~mA}$, the voltage output is $75 \mathrm{~V}$, the pulse interval is 5 30s, and the wave width is 50 100ms. The model was successfully constructed by stimulating rats to achieve systolic blood pressure of $150 \mathrm{~mm} \mathrm{Hg}$ after 15 days.

2.3 Measurement of blood pressure and heart rate of rats and RVLM injection

(1) Preparation before the operation: after weighing, intraperitoneal injection of pentobarbital sodium $(40 \mathrm{mg} / \mathrm{kg})$, the rats were fixed on the operating table and intubated with endotracheal intubation and right femoral arteriovenous intubation. Blood pressure and heart rate were monitored using a pressure sensor connected to a biometric recording system. The animal is placed in the prone position with its head fixed on the stereotactic locator. Endotracheal intubation was connected with animal ventilator, and $1 \%$ triiodoquaternary phenol $(10 \mathrm{mg} / \mathrm{kg}, 3 \mathrm{mg} / \mathrm{kg}$ injection every $0.5 \mathrm{~h})$ was injected into femoral vein to brake. Cut the craniotomy top to the back skin along the midline, remove part of occipital bone and cerebellum, fully expose the medulla oblongata, and adjust the medulla oblongata to the horizontal position. Urethane supplementation $(0.4 \mathrm{~g} / \mathrm{kg})$ was used to maintain appropriate anesthesia throughout the operation. The anal temperature of the rats was maintained at around $37^{\circ} \mathrm{C}$ by infrared lamp irradiation.

(2) RVLM injection: after the operation, the rats were administered after the vital signs were stabilized for $3 \mathrm{~min}$. According to the stereotypic mapping of rat brain [8], the coordinates take the bar of medulla oblongata as the origin. RVLM is $2.5-2.9 \mathrm{~mm}$ 
to the end, $1.6-2.0 \mathrm{~mm}$ to the side, and the depth of penetration is $3-3.4 \mathrm{~mm}$.Melatonin or artificial cerebrospinal fluid (CSF) were injected into three self-made glass microelectrodes (20 30 cm outside the tip).The three microelectrodes were installed on the micromanipulator for injection. The volume of drug injection was $100 \mathrm{~nL}$ and the injection time was 5-10s.

(3) RVLM identification: bilateral microinjection of glutamate ( $2 \mathrm{nmol})$ caused a rapid increase in systolic blood pressure of 20-30mmhg. The dose of both side injection drugs was $50 \mathrm{~nL}$, and the injection was completed within $1 \mathrm{~min}$. Before the end of the experiment, 50nL Evans blue was injected into both RVLM to identify the injection site. After the rats were anesthetized, their brains were taken out and soaked in $10 \%$ formalin solution for a week. The injection site was identified by brain biopsy. Experimental data that were not at the injection site in the RVLM area were not statistically processed

2.4 Detection of glutamate, a-aminobutyric acid and norepinephrine expression in RVLM tissues by ELISA

RVLM tissues of each group were taken under the ice bath, and the ultrasonic lysis tissues were taken under the ice bath for 30s.Centrifuge at low temperature for $30 \mathrm{~min}$ at $4^{\circ} \mathrm{C}$, with a speed of $14000 \mathrm{r} / \mathrm{min}$. After centrifugation, the supernatant was extracted with $0.22 \mathrm{~m}$ filter membrane, and the filtrate was used as the sample solution. Under the ice bath, $5 \mathrm{ml}$ of rat venous blood was taken, and after anticoagulation with heparin, $1500 \mathrm{~g}$ centrifugal force was used for $5 \mathrm{~min}$ to separate the plasma. The expression of glutamate, p-aminobutyric acid and plasma norepinephrine in RVLM tissues was detected by GeneChem (Shanghai, PRC).

2.5 Detection of the expression of CD86, TNF-, il-1 and c-fos mRNA in RVLM tissues by real-time PCR

Total RNA was extracted from the RNAeasy ${ }^{\mathrm{TM}}$ cellular RNA extraction kit (centrifugal column). The target protein and internal reference primers were synthesized by Sangon (Shanghai, PRC). The reaction Kit of Quant one step qRT-PCR Kit was used, and the reaction system and amplification conditions were configured as recommended by the Kit instructions. PCR amplification was performed on ABI7700 real-time quantitative PCR instrument. The program automatically calculated the ratio of PCR product of target gene mRNA to internal 
reference GAPDH absorbance value as the relative expression of target gene. The PCR primers used were as follows:

CD86: 5'-AATCCTTTTCTCGGTGTTGG-3' (sense) and

5'-CTCGGGCTTATGTTTTGAGC-3' (anti-sense); TNF- $\alpha$ :

5'-ACAAGGCTGCCCCGACTAT-3' (sense) and

5'-CTCCTGGTATGAAGTGGCAAATC-3' (anti-sense); IL-1 3 :

5'-CACCTCTCAAGCAGAGCACAG-3' (sense) and

5'-GGGTTCCATGGTGAAGTCAAC-3' (anti-sense); c-fos: 5'-ACCATGATGT

TCTCGGGTTTCAAC-3' (sense) and 5'-GAGATGGCTGTCACCGTGGGGAT-3'

(anti-sense).

2.6 Statistics analysis

All data are presented as mean \pm standard error of the mean (SEM). For experiments that involved two groups of samples, Student's unpaired $t$ test was used. For experiments that involved multiple groups, one-way or two-way analysis of variance with repeated measures were used to assess group means.

\section{Results}

3.1 RVLM injection of melatonin reduced blood pressure and reduced plasma NE concentration in SIH rats

The systolic blood pressure of the SIH rats was $176 \mathrm{mmHg}( \pm 6 \mathrm{mmHg})$, significantly higher than that of the control group [112mmHg $\pm 4 \mathrm{mmHg}, \mathrm{P}<0.05]$. RVLM injection of melatonin significantly reduced blood pressure in SIH rats, and the hypotensive effect was concentration dependent $(\mathrm{p}<0.05)$, while artificial cerebrospinal fluid injection had no significant effect on blood pressure in SIH rats (Figure 1). The plasma NE level of SIH rats was significantly higher than that of control group $(\mathrm{p}<0.05)$. Consistent with the effect of melatonin injection by RVLM on blood pressure of SIH rats, different concentrations of melatonin significantly reduced the plasma NE concentration of SIH rats, which also showed a concentration gradient dependence (Figure 2).

3.2 Melatonin inhibited M1 polarization and inflammatory factor release in RVLM microglia in SIH rats

The expression of M1 polarization marker protein CD86 in SIH rats RVLM 
microglia was significantly increased compared with control group $(\mathrm{p}<0.05)$. RVLM injection of melatonin significantly decreased the expression of CD86 with a concentration dependent effect (all p<0.05), while artificial cerebrospinal fluid injection had no significant effect on the expression of CD86 in SIH rats (Figure 3).

The expression of RVLM inflammatory factors in SIH rats was significantly higher than that in $\mathrm{Ctrl}$ group $(\mathrm{p}<0.05)$. Consistent with the effect of melatonin injection by RVLM on the expression of inflammatory factors in SIH rats, melatonin at different concentrations significantly reduced the expression of inflammatory factors in RVLM in SIH rats, and the concentration was also gradient dependent (Figure 4).

3.3 Melatonin decreased the expression of excitatory neurotransmitter glutamate and c-fos expression in stress-induced RVLM rats, and increased the expression of inhibitory neurotransmitter-aminobutyric acid

The expression of excitatory neurotransmitter glutamate in SIH rats was significantly increased compared with control group $(\mathrm{p}<0.05)$, while the expression of inhibitory neurotransmitter GABA was not changed. Melatonin injection into RVLM significantly decreased glutamic acid expression and increased GABA expression, both of which were concentrate-dependent $(\mathrm{p}<0.05)$, while artificial cerebrospinal fluid injection had no significant effect on glutamate and GABA expression in SIH rats (Figure 5-6). The c-fos expression in RVLM of SIH rats was significantly higher than that in the control group $(\mathrm{p}<0.05)$. Consistent with the effect of melatonin injection by RVLM on glutamic acid expression in SIH rats, all test concentrations of melatonin significantly reduced the expression of c-fos in SIH rats, which also showed a concentration gradient dependence (Figure 7).

\section{Discussion}

The pathogenesis of hypertension is complicated, such as heart rate, cardiac output and total peripheral resistance. With the deepening understanding of the pathogenesis of neurogenic hypertension, it has been found that the pathogenesis of hypertension is closely related to environmental stress, mental tension, sympathetic excitability, neurological dysfunction and other mechanisms. The central nervous system and sympathetic nerves play an important role in the regulation of blood pressure. In both humans and animals, changes in stress response regulation of the 
hypothalamic-pituitary-adrenal axis, sympathetic adrenal medulla system, renin-angiotensin-aldosterone system, and sympathetic nervous system can occur under certain stress [9]. The regulation of sinoatrial baroreceptors and peripheral blood vessels, as well as the significant increase of catecholamines in blood and urine, cause the continuous increase of blood pressure. Chronic social psychological stress, emotion, sound, light, electricity, cold, wind and other stimuli are important causes of SIH $[10,11]$.

RVLM is the most basic cardiovascular center, and its local renin-angiotensin system (RAS) is involved in the occurrence of hypertension independently of circulating RAS, and the complexity of its regulation of central hypertension is related to the pro-inflammatory factor mechanism dependent on glial cells. Microglial activation is a marker of neuroinflammation. It has been confirmed that RVLM "neuroinflammation-sympathetic activation" is involved in the formation of SIH. We and other researchers have reported that neuroinflammation, microglial activation and oxidative stress in the RVLM region are involved in SIH regulation [13, 14]. Microglia are immune cells in the brain. When activated at rest, microglia (M0) present two phenotypes, M1 and M2. The transformation of M0 microglia cells into these two phenotypes is called microglia polarization.M1-type is classically activated, releasing inflammatory cytokines including cytotoxic substances such as nitric oxide (NO), oxygen free radicals, proteolytic enzymes, and IL- $1 \beta$, TNF- $\alpha$, IFN- $\gamma$, which are associated with neurotoxicity. The M2-type is the alternative activated type, which can secrete anti-inflammatory factors such as TGF- $\beta$ and IL-10, and has the function of inhibiting inflammatory response and secreting neurotrophic factor, which is related to the repair and regeneration of nerve tissue. During the process of maintaining microenvironmental homeostasis, there was a dynamic balance between M1 and M2 microglia. Li et al [15] reported that mental abnormalities were associated with microglia polarization: compared with mice with mild anxiety, microglia in mice with severe anxiety were more polarized into M1-type. This suggests that the polarization phenotype of microglia is related to the degree of stress. Some scholars believe that the occurrence of cardiovascular diseases, such as hypertension, may be related to the loss of microglia cells' homeostasis in maintaining the physiological activities of sympathetic neurons in the cardiovascular center [16].

The pineal gland was called the "biological timer" because melatonin secretion was associated with the body's aging process. Melatonin is widely found in various 
organs of the body. It is a hormone with multiple functions, including promoting sleep, anti-aging, immune regulation, neuroprotection, anti-tumor, other endocrine regulation, and promoting cell growth. Previous studies have shown that abnormal blood pressure circadian rhythm is significantly correlated with target organ damage and cardiovascular events, and is an important risk factor independent of blood pressure level [17]. Melatonin has anti-inflammatory, antioxidant and hypotensive effects. Nocturnal hypertension and gestational hypertension can cause higher mortality and mortality. Numerous studies have found that patients with high blood pressure levels have relatively low melatonin levels in the body than normal and taking melatonin can help reduce blood pressure in patients with hypertension, because melatonin can act on peripheral blood endodermal cells, exert vasodilatory, antioxidant, free radical scavenging, improve diastolic function, regulate metabolism and anti-inflammatory effects. Melatonin plays an important role in protecting the damaged vascular endothelial cells, thereby reducing blood pressure, which is the peripheral mechanism of melatonin in lowering blood pressure [18-20]. However, the central antihypertensive mechanism of melatonin remains unclear.

A large number of studies have shown that melatonin has two types of receptors, namely melatonin receptor 1 (MLT1) and melatonin receptor 2 (MLT2). Melatonin excited specific receptor activates the corresponding second signal transduction system in the cell, and then influences the expression of neurotrophic factor (BDNF) through the third messenger in the nucleus, such as cAMP response element binding protein (CREB), thereby changing the function of nerve cells and playing a regulatory role in sleep, biological rhythm and mood. In recent years, many researchers use agonists and blockers to regulate the two subtype receptors of MLT1 and MLT2, and the results of their pharmacological properties are also different. Neu-p11, a novel melatonin receptor agonist, has antihypertensive effects in spontaneously hypertensive rats, which may be related to promoting the synthesis and release of NO in serum and reducing the content of serum ET-1 [21]. As the body ages, the thymus gland atrophies, resulting in a decrease in the number of $\mathrm{T}$ lymphocytes. The effect of melatonin on immune regulation is mainly to increase the weight of the thymus gland, enhance the activity of helper $\mathrm{T}$ cells, promote the proliferation of $\mathrm{T}$ lymphocytes and B lymphocytes, and further enhance the toxicity of natural killer cells [22, 23]. Melatonin promotes both innate and acquired immunity. However, it is not clear whether melatonin directly regulates the cytokines releasing from microglia cells, thus 
resulting in the mechanism of immune regulation. Studies have shown that the active substances produced by the vascular endothelial system in body fluids, such as endothelin, nitric oxide and angiotensin aldosterone system (RAAS), show significant circadian rhythm changes and are significantly correlated with the circadian rhythm of blood pressure. In patients with hypertension, the changes of these humoral factors may be the pathological basis of abnormal blood pressure rhythm. Previous studies have shown that melatonin can reduce blood pressure by affecting vascular endothelial system and RAAS system [24, 25], but the mechanism is not completely clear. In this study, the stress-induced hypertension rat model was adopted to observe the antihypertensive effect of melatonin, and the central antihypertensive mechanism and target of melatonin were explored based on the neuroinflammation triggered by M1 polarization of microglia cells, providing experimental basis for further clarifying the mechanism of melatonin's involvement in regulating blood pressure.

\section{Conclusions}

We found that RVLM injection of melatonin can inhibit microglia-mediated neuroinflammation caused by stress, block "neuroinflammation-sympathetic activation". Our research may provide an experimental basis for the prevention and treatment of stress hypertension.

\section{Data Availability}

All relevant data are within the manuscript and figures.

\section{Conflicts of Interest}

The author declares that the research was conducted in the absence of any commercial or financial relationships that could be construed as a potential conflict of interest.

\section{Funding Statement}

This work was supported by the "Qing Feng" Undergraduate Innovation and Entrepreneurship Scholarship of Shanghai Medical college, Fudan University (QF1703) to STZ. The funders had no role in study design, data collection and analysis, decision to publish, or preparation of the manuscript.

\section{Acknowledgments}

We sincerely thank $\mathrm{Li} \mathrm{Hu}$ M.S. of Laboratory of Neuropharmacology and 
Neurotoxicology in Shanghai University for providing technical assistance.

\section{Reference:}

1. Oparil S, Zaman MA, Calhoun DA. Pathogenesis of hypertension. Ann Intern Med. 2003 Nov 4;139(9):761-76. Review.

2. Fisher JP, Fadel PJ. Therapeutic strategies for targeting excessive central sympathetic activation in human hypertension. Exp Physiol. 2010 May;95(5):572-80.Delneste Y, Magistrelli G, Gauchat J, et al. Involvement of LOX-1 in dendritic cell-mediated antigen cross-presentation [J]. Immunity, 2002,17(3): 353-362.

3. Labandeira-Garcia J L , Rodríguez-Perez Ana I, Pablo G G , et al. Brain Renin-Angiotensin System and Microglial Polarization: Implications for Aging and Neurodegeneration[J]. Frontiers in Aging Neuroscience, 2017, 9:129-.

4. Wang LH, Dong T, Liu BB, Zhao XD, Chen JW, Murao K, Zhu W, Zhang GX. Contribution of the renin-angiotensin system in chronic foot-shock induced hypertension in rats. Life Sci. 2015 Jan 15;121:135-44.

5. Holmes S W, Sugden D. Proceedings: The effect of melatonin on pinealectomy-induced hypertension in the rat $[\mathrm{J}]$. British Journal of Pharmacology, 1976, 56(3):360P.

6. Reiter R J, Tan D X, Korkmaz A. The circadian melatonin rhythm and its modulation: possible impact on hypertension.[J]. J Hypertens Suppl, 2009, 27(6):S17.

7. Xia C M, Shao C H, Xin L, et al. Effects of melatonin on blood pressure in stress-induced hypertension in rats. Clin Exp Pharmacol Physiol 2008 Clinical \& Experimental Pharmacology \& Physiology, 2008, 35(10):1258-1264.

8. Paxinos G, 1944-, Watson C, et al. rat brain in stereotaxic coordinates[J]. 1997.

9. Hering D, Lachowska K, Schlaich M. Role of the Sympathetic Nervous System in Stress-Mediated Cardiovascular Disease. Curr Hypertens Rep. 2015 Oct; 17(10):80.

10. Allgulander C. Anxiety as a risk factor in cardiovascular disease. Curr Opin Psychiatry. 2016 Jan;29(1):13-17;Psychosocial stress and cardiovascular risk:what 
is the role of daily experience? J Pers,2005 Dec,73(6):1749—74.

11. Kumagai H, Oshima N, Matsuura T, ligaya K, Imai M, Onimaru H, Sakata K, Osaka M, Onami T, Takimoto C, Kamayachi T, Itoh H, Saruta T. Importance of rostral ventrolateral medulla neurons in determining efferent sympathetic nerve activity and blood pressure. Hypertens Res. 2012 Feb;35(2):132-41.

12. Zhang CR, Xia CM, Jiang MY, Zhu MX, Zhu JM, Du DS, Liu M, Wang J, Zhu DN. Repeated electroacupuncture attenuating of apelin expression and function in the rostral ventrolateral medulla in stress-induced hypertensive rats. Brain Res Bull, 2013, 97: 53-62.

13. Du D, Hu, Li, Wu, Jiaxiang, et al. Neuroinflammation contributes to autophagy flux blockage in the neurons of rostral ventrolateral medulla in stress-induced hypertension rats[J]. Journal of Neuroinflammation, 2017, 14(1):169..

14. Shi P, Diez-Freire, C, Jun, J. Y, et al. Brain Microglial Cytokines in Neurogenic Hypertension[J]. Hypertension, 2010, 56(2):297-303.

15. Li Z, Ma L, Kulesskaya N, Võikar V, Tian L. Microglia are polarized to M1 type in high-anxiety inbred mice in response to lipopolysaccharide challenge. Brain Behav Immun. 2014 May;38:237-48.

16. Alerted microglia and the sympathetic nervous system: A novel form of microglia in the development of hypertension. Respir Physiol Neurobiol. 2015 Nov 28. pii: S1569-9048(15)30087-2.

17. Perloff D. Retrospective and prospective research on hypertension-related end-organ damage[J]. Journal of Cardiovascular Pharmacology, 1994, 24 Suppl A(24 Suppl A):S1.

18. Grossman E, Moshe Laudon, Ridvan Yalcin, et al. Melatonin Reduces Night Blood Pressure in Patients with Nocturnal Hypertension[J]. American Journal of Medicine, 2006, 119(10):898-902.

19. You-Lin Tain, Li-Tung Huang, Julie Y. H. Chan. Transcriptional Regulation of Programmed Hypertension by Melatonin: An Epigenetic Perspective[J]. International Journal of Molecular Sciences, 2014.

20. Paulis L, Pechanova, Olga, Zicha, Josef, et al. Melatonin improves the restoration of endothelium-derived constricting factor signalling and inner diameter in the rat 
femoral artery after cessation of L-NAME treatment[J]. Journal of Hypertension, 2010, 28(9):S19-24.

21. She M, Xiaojian Deng, Zhenyu Guo, et al. NEU-P11, a novel melatonin agonist, inhibits weight gain and improves insulin sensitivity in high-fat/high-sucrose-fed rats[J]. Pharmacological Research the Official Journal of the Italian Pharmacological Society, 2009, 59(4):248-253.

22. Carrillo-Vico A, Lardone, Patricia, álvarez-Sánchez, Nuria, et al. Melatonin: Buffering the Immune System[J]. International Journal of Molecular Sciences, 2013, 14(4):8638-8683.

23. Reiter R J, Calvo J R, Karbownik M, et al. Melatonin and its relation to the immune system and inflammation[J]. Ann N Y Acad Sci, 2010, 917(1):376-386.

24. Simko F, Baka T, Krajcirovicova K, et al. Effect of Melatonin on the Renin-Angiotensin-Aldosterone System in 1-NAME-Induced Hypertension[J]. Molecules, 2018, 23(2):265.

25. Tamura E K, Silva C L, Markus R P. Melatonin inhibits endothelial nitric oxide production in vitro[J]. Journal of Pineal Research, 2010, 41(3):267-274. 


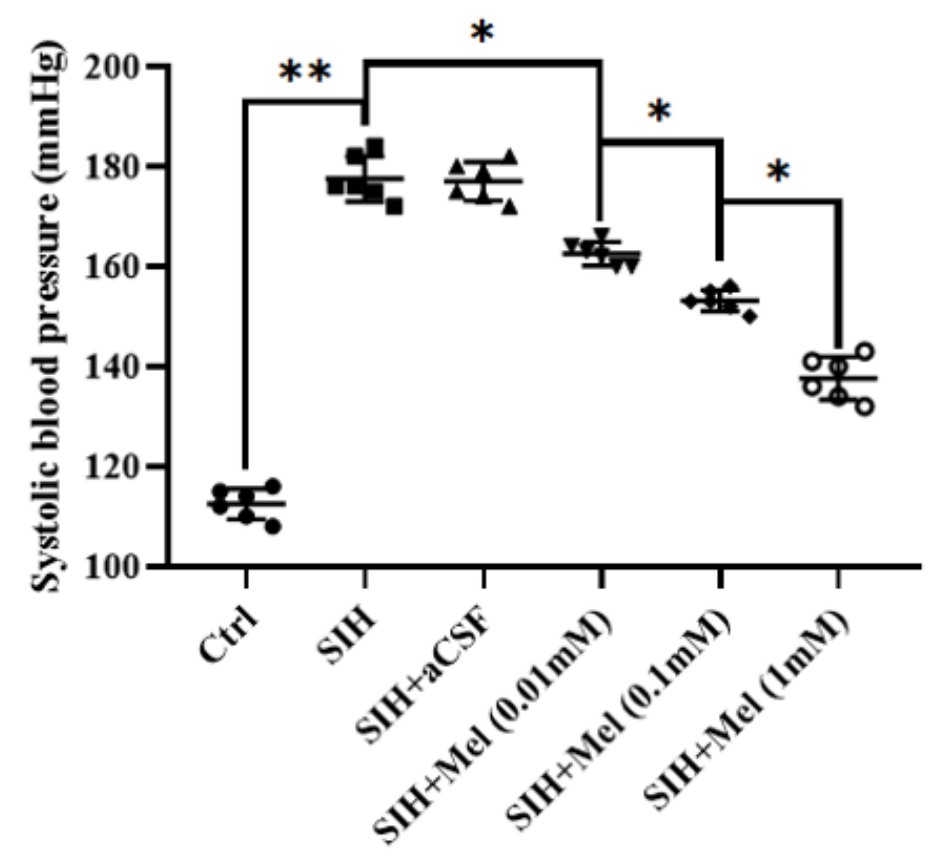

Figure 1. Effects of RVLM injection of melatonin at different concentrations on systolic blood pressure in SIH rats. The systolic blood pressure of SIH rats was significantly higher than that of control group. RVLM injection of melatonin significantly reduced blood pressure in SIH rats, and the hypotensive effect was concentration dependent. However, artificial cerebrospinal fluid injection had no significant effect on blood pressure of SIH rats. $\left(n=6,{ }^{*} p<0.05, * * p<0.01\right)$ 


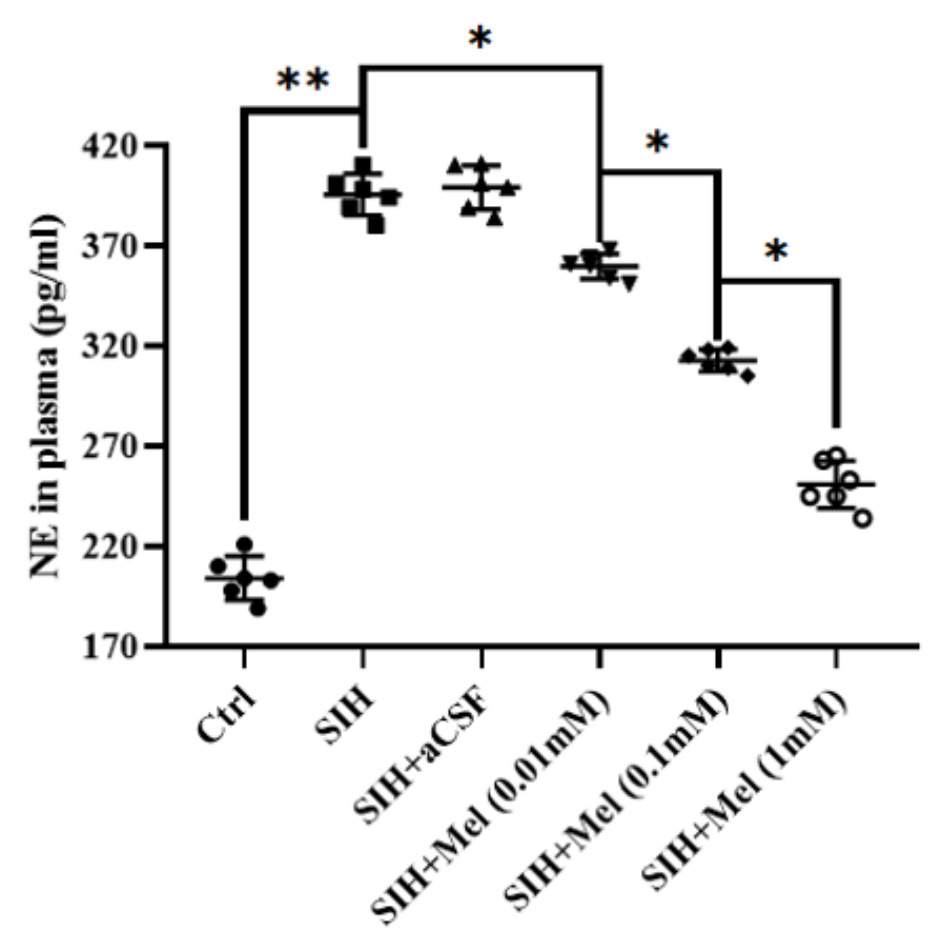

Figure 2. Effects of RVLM injection of melatonin at different concentrations on plasma $\mathrm{NE}$ concentration in SIH rats. The plasma NE concentration of SIH rats was significantly higher than that of control group. RVLM injection of melatonin significantly reduced the plasma NE concentration in SIH rats, and the effect was concentration dependent. However, injection of artificial cerebrospinal fluid (CSF) had no significant effect on plasma NE concentration in SIH rats. $(\mathrm{n}=6, * \mathrm{p}<0.05, * * \mathrm{p}<0.01)$ 


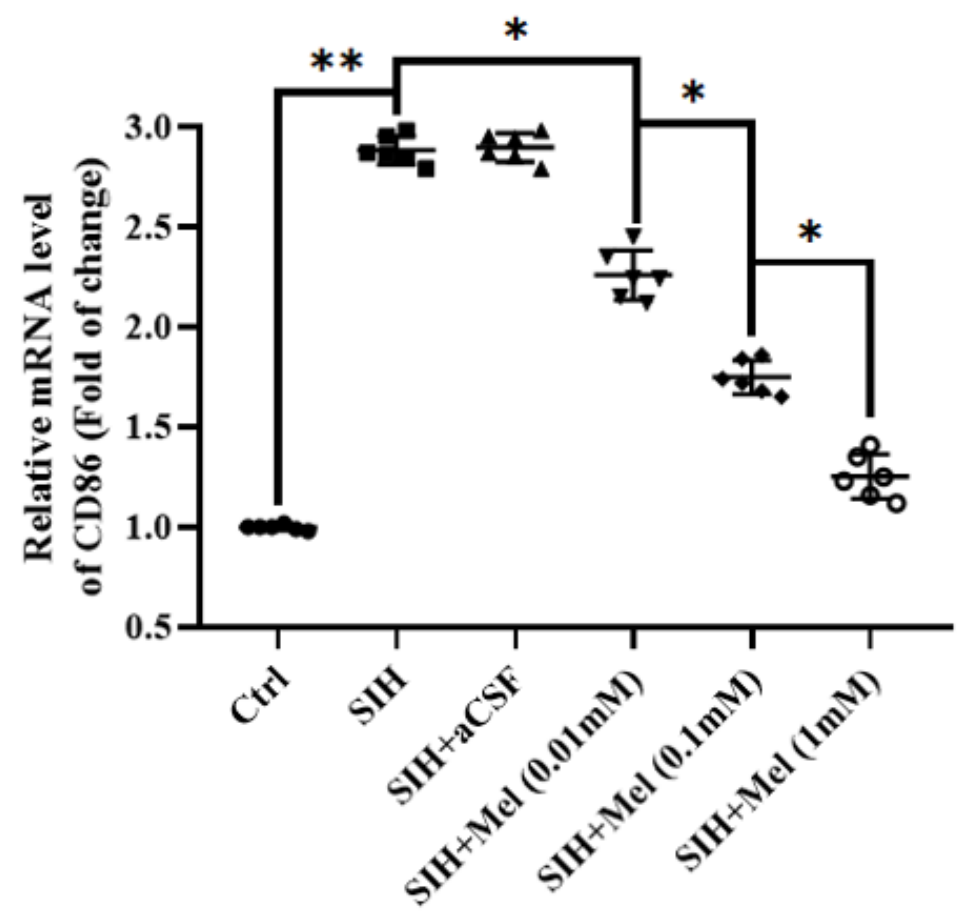

Figure 3. Effects of different concentrations of melatonin on CD86 expression in RVLM tissues in SIH rats after injection of RVLM. The expression of CD86 in RVLM of SIH rats was significantly higher than that in control rats.Injection of melatonin into RVLM significantly reduced the expression of CD86 in RVLM of SIH rats, and the effect was concentration-dependent.However, injection of artificial cerebrospinal fluid had no significant effect on CD86 expression in RVLM of SIH rats. $\left(\mathrm{n}=6,{ }^{*} \mathrm{p}<0.05, * * \mathrm{p}<0.01\right)$ 


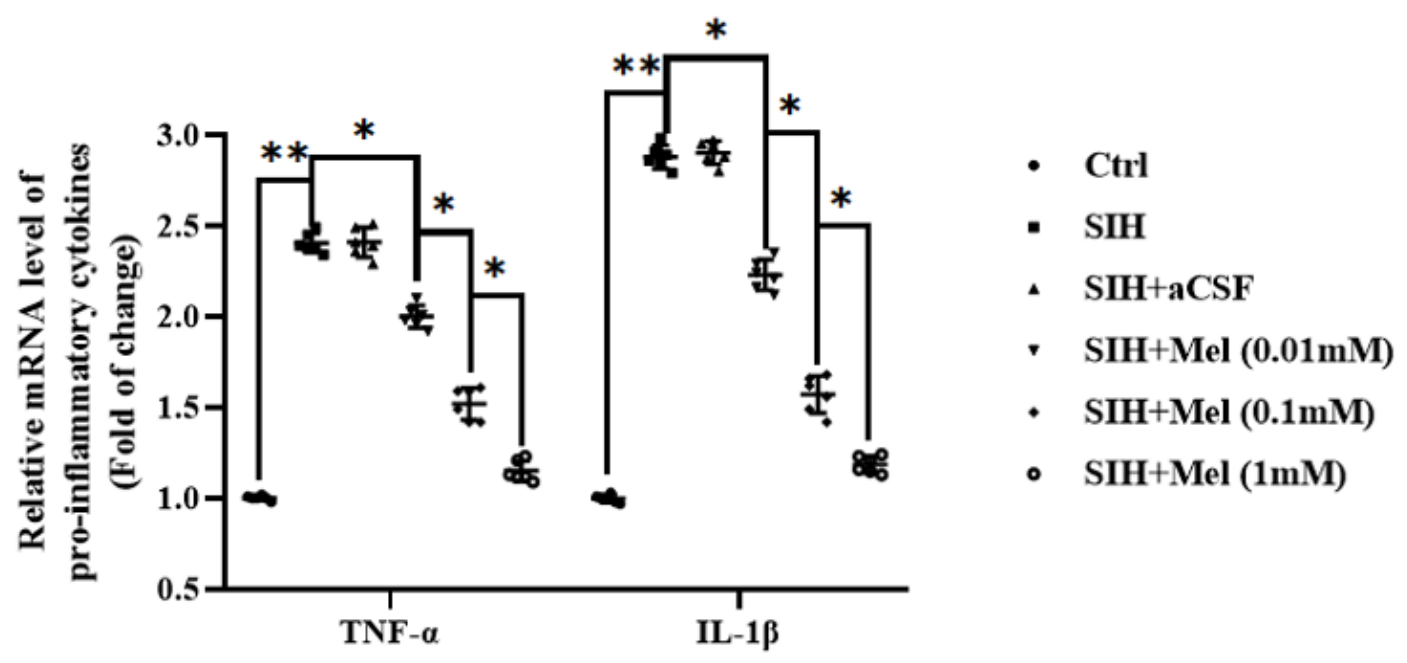

Figure 4. Effects of different concentrations of melatonin on the expression of inflammatory factors in RVLM tissues of SIH rats injected with RVLM. The expression of inflammatory factors in RVLM tissues of SIH rats was significantly higher than that of control rats. Injection of melatonin into RVLM significantly reduced the expression of inflammatory factors in RVLM tissues of SIH rats, and the effect was concentration dependent. However, the injection of artificial cerebrospinal fluid had no significant effect on the expression of inflammatory factors in RVLM tissues of SIH rats. $(n=6, * p<0.05, * * p<0.01)$ 


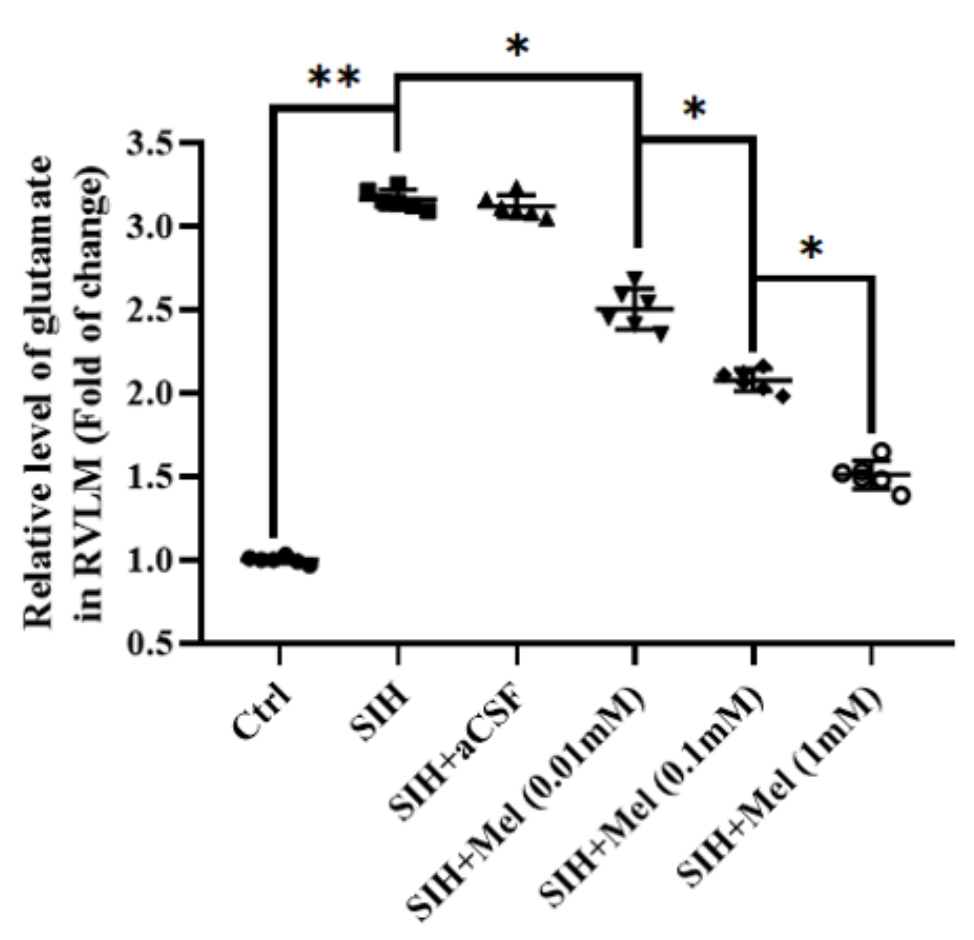

Figure 5. Effects of different concentrations of melatonin on glutamate expression of RVLM in SIH rats. The expression of glutamate in RVLM tissues of SIH rats was significantly higher than that of control rats. Melatonin injection into RVLM significantly reduced glutamate expression in RVLM tissues of SIH rats, and the effect was concentration dependent. However, injection of artificial cerebrospinal fluid had no significant effect on glutamate expression in RVLM tissues of SIH rats. $(\mathrm{n}=6, * \mathrm{p}<0.05, * * \mathrm{p}<0.01)$ 


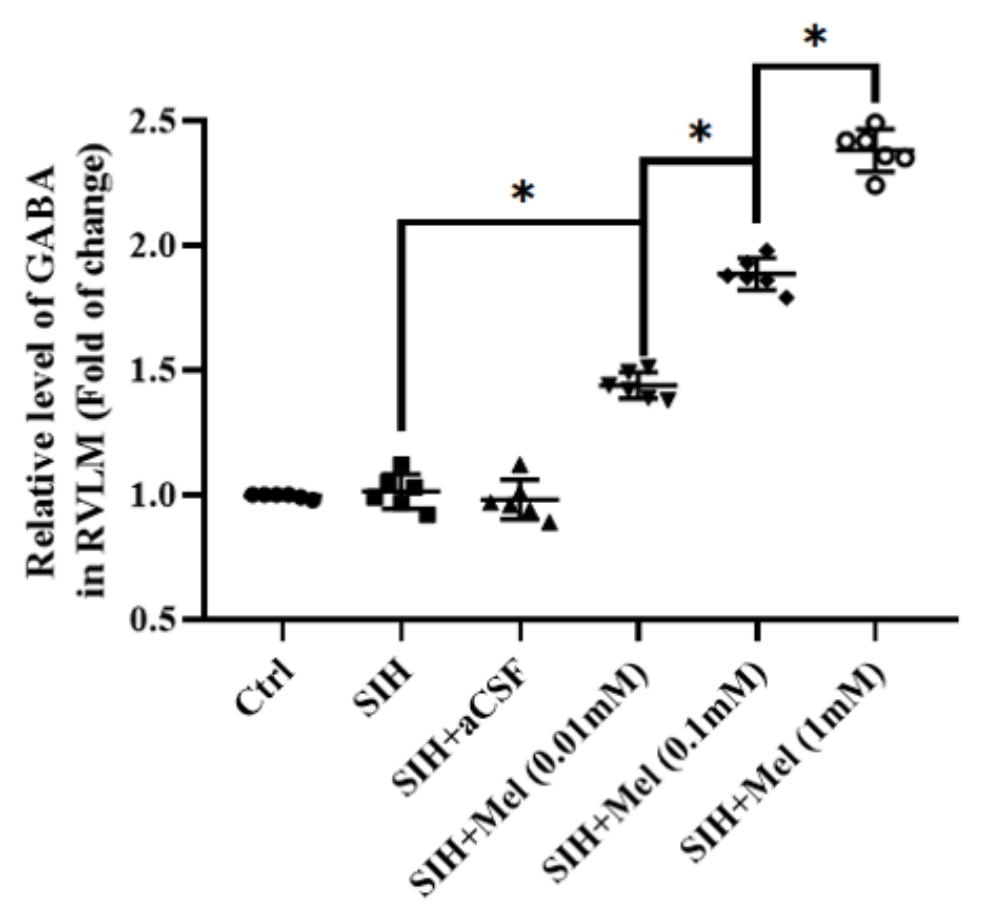

Figure 6. Effects of different concentrations of melatonin on the expression of RVLM aminobutyric acid in SIH rats injected with RVLM. The expression of amino butyric acid in RVLM tissues of SIH rats was significantly higher than that of control rats. Injection of melatonin into RVLM significantly reduced the expression of RVLM amino butyric acid in SIH rats, and the effect was concentration dependent. However, the injection of artificial cerebrospinal fluid had no significant effect on the expression of RVLM -aminobutyric acid in RVLM tissues of SIH rats. $\left(n=6,{ }^{*} p<0.05, * * p<0.01\right)$ 


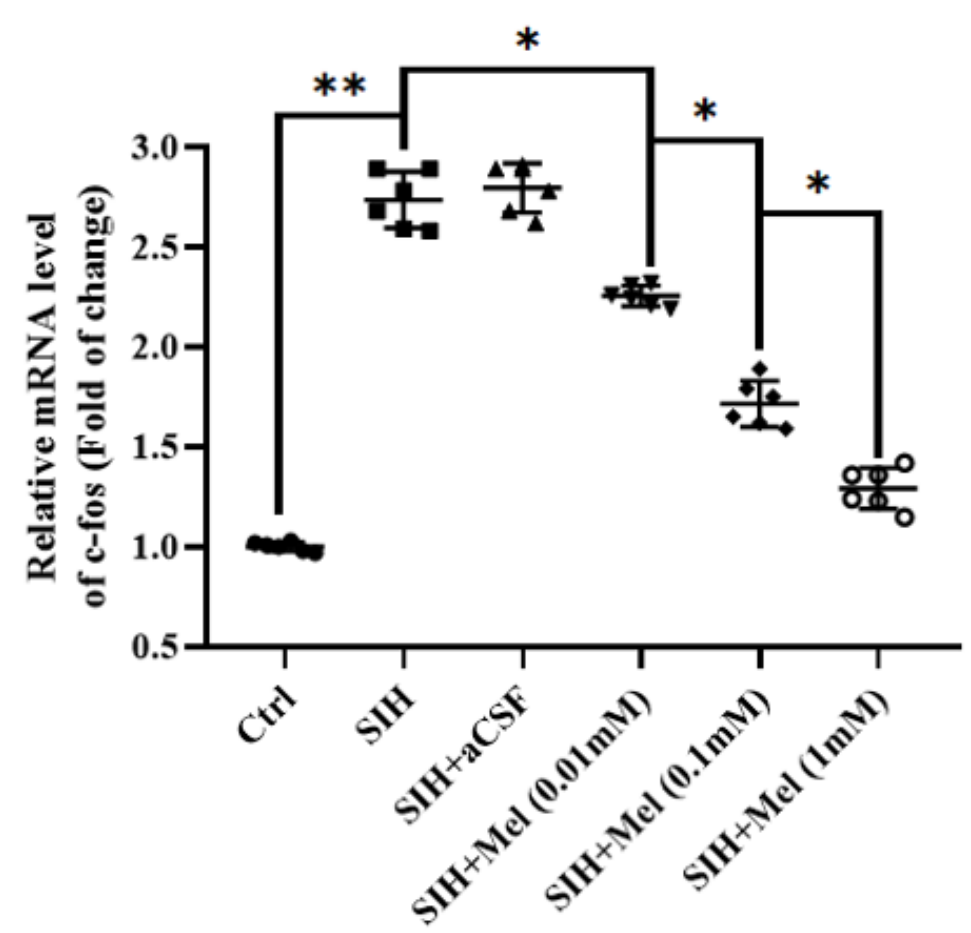

Figure 7. Effects of different concentrations of melatonin on the expression of c-fos in RVLM in SIH rats. The expression of c-fos in RVLM immediate early gene in SIH rats was significantly higher than that in control rats. Injection of melatonin into RVLM significantly reduced the expression of c-fos in the early gene of RVLM in SIH rats, and the effect was concentration dependent. However, injection of artificial cerebrospinal fluid (CSF) had no significant effect on the expression of RVLM gene $\mathrm{c}-\mathrm{fos}$ in SIH rats. $\left(\mathrm{n}=6,{ }^{*} \mathrm{p}<0.05, * * \mathrm{p}<0.01\right)$ 